\title{
How atomic nuclei cluster
}

\author{
J.-P. Ebran, ${ }^{1}$ E. Khan, ${ }^{2}$ T. Niksic, ${ }^{3}$ and D. Vretenar ${ }^{3}$ \\ ${ }^{1}$ CEA/DAM/DIF, F-91297 Arpajon, France \\ ${ }^{2}$ Institut de Physique Nucléaire, Université Paris-Sud, IN2P3-CNRS, F-91406 Orsay Cedex, France \\ ${ }^{3}$ Physics Department, Faculty of Science, University of Zagreb, 10000 Zagreb, Croatia
}

(Dated: November 1, 2018)

\begin{abstract}
Nucleonic matter displays a quantum liquid structure, but in some cases finite nuclei behave like molecules composed of clusters of protons and neutrons. Clustering is a recurrent feature in light nuclei, from beryllium to nickel. For instance, in ${ }^{12} \mathrm{C}$ the Hoyle state, crucial for stellar nucleosynthesis, can be described as a nuclear molecule consisting of three alpha-particles. The mechanism of cluster formation, however, has not yet been fully understood. We show that the origin of clustering can be traced back to the depth of the confining nuclear potential. By employing the theoretical framework of energy density functionals that encompasses both cluster and quantum liquid-drop aspects of nuclei, it is shown that the depth of the potential determines the energy spacings between single-nucleon orbitals, the localization of the corresponding wave functions and, therefore, the degree of nucleonic density clustering. Relativistic functionals, in particular, are characterized by deep single-nucleon potentials. When compared to non-relativistic functionals that yield similar ground-state properties (binding energy, deformation, radii), they predict the occurrence of much more pronounced cluster structures. More generally, clustering is considered as a transitional phenomenon between crystalline and quantum liquid phases of fermionic systems.
\end{abstract}

The occurrence of molecular states in atomic nuclei and the formation of clusters of nucleons were already predicted in the 30's by von Weizsäcker and Wheeler [1, 2]. Even though the description of nuclear dynamics became predominantly based on the concept of independent nucleons in a mean-field potential, a renewed interest in clustering phenomena in the 60's led to the development of dedicated theoretical methods 3 . Numerous experimental studies have revealed a wealth of data on clustering phenomena in light nuclei [4, and modern theoretical approaches use microscopic models that fully take into account single-nucleon degrees of freedom [5] 7]. Clustering gives rise to nuclear molecules. For instance, in ${ }^{12} \mathrm{C}$ the second $0^{+}$state the Hoyle state that plays a critical role in stellar nucleosynthesis, is predicted to display a three- $\alpha$ structure 8,9 . The binding energy of the $\alpha$-particle, formed from two protons and two neutrons, is much larger than in other light nuclei. Cluster radioactivity [10], discovered in the 80's, is another manifestation of clustering in atomic nuclei. Experimental signatures of clustering are usually indirect. Quasi-molecular resonances are probed by scattering one cluster on another, such as in the ${ }^{12} \mathrm{C}+{ }^{12} \mathrm{C}$ system [4, 11], and cluster structures are also discernible in the breakup of nuclei. Evidence has been reported for the formation of clusters in ground and excited states of a number of $\alpha$-conjugate nuclei [4, that is nuclei with an equal even number of protons and neutrons, from ${ }^{8} \mathrm{Be}$ to ${ }^{56} \mathrm{Ni}$.

The mechanism of cluster formation in nuclei has not yet been fully understood. Deformation plays an important role because it removes the degeneracy of singlenucleon levels associated with spherical symmetry. At specific deformations the shell structure can restore degeneracies corresponding, for instance, to a 2:1 ratio of the large axis over the small axis of a quadrupole deformed system4. Consequently, the restored degener- acy of deformed shell closures facilitates the formation of clusters. However, this is a rather qualitative explanation because clustering phenomena cannot generally be explained by accidental degeneracies. Clustering is an essential feature of many-nucleon dynamics that coexists with the nuclear mean-field. Therefore, although in most cluster models the existence of such structures is assumed a priori and the corresponding effective interactions are adjusted to the binding energies and scattering phase shifts of these configurations, a fully microscopic understanding of cluster formation necessitates a more general description that encompasses both cluster and quantum liquid-drop aspects in light as well as in heavier nuclei. The aim of this work is to address the origin of clustering, i.e. to find out the conditions for cluster formation in ground states of finite nuclei, starting from a fully microscopic description based on the framework of energy density functionals.

At present the only comprehensive approach to nuclear structure is based on the framework of energy density functionals (EDFs). Nuclear EDFs enable a complete and accurate description of ground-state properties and collective excitations over the whole nuclide chart 12 14. In practical implementations nuclear EDFs are analogous to Kohn-Sham Density Functional Theory, the most widely used method for electronic structure calculations in condensed matter physics and quantum chemistry. In the nuclear case the many-body dynamics is represented by independent nucleons moving in a local self-consistent mean-field potential that corresponds to the actual density and current distribution of a given nucleus. Both non-relativistic and relativistic realizations of EDFs are employed in studies of nuclear matter and finite nuclei. A nuclear EDF is universal in the sense that, for a given inter-nucleon interaction, it has the same functional form for all systems. Using a small set of global 
parameters adjusted to empirical properties of homogeneous nuclear matter and data on finite nuclei, a universal functional provides a description of the structure of nuclei across the chart of nuclides and, therefore, describes the coexistence of cluster and quantum-liquid aspects of light nuclei.

A number of recent studies based on nuclear EDFs or the mean-field approach have analysed cluster structures in $\alpha$-conjugate nuclei 15 21. In Fig. 1 we display the self-consistent ground-state densities of ${ }^{20} \mathrm{Ne}$, calculated with two widely used functionals that are representative for the two classes of nuclear EDFs: the non-relativistic Skyrme SLy4 [22, and the relativistic functional DDME2 [23]. The equilibrium shape of ${ }^{20} \mathrm{Ne}$ is a prolate, axially symmetric quadrupole ellipsoid. Although they have not been specifically adjusted to this mass region, both functionals reproduce the empirical ground-state properties of this nucleus: the experimental binding energy $160.6 \mathrm{MeV}$, the radius of the proton distribution 2.90 fm [24], and the radius of the matter distribution 2.85 fm [25], with a typical accuracy of $1 \%$. It is remarkable that, although these functionals predict similar values for the binding energy, charge and matter radii, as well as the quadrupole deformation of equilibrium shape of ${ }^{20} \mathrm{Ne}$, yet the corresponding single-nucleon densities are qualitatively very different. The density calculated with SLy4 displays a smooth behaviour characteristic for a Fermi liquid, with an extended surface region in which the density very gradually decreases from the central value of $0.16 \mathrm{fm}^{-3}$. The relativistic functional DD-ME2, on the other hand, predicts an equilibrium density that is much more localized. The formation of cluster structures is clearly visible, with density spikes as large as $0.2 \mathrm{fm}^{-3}$, and a much narrower surface region.

Understanding the difference in the equilibrium densities of ${ }^{20} \mathrm{Ne}$ calculated with SLy4 and DD-ME2 is the key to the mechanism of cluster formation in this mass region of $\alpha$-conjugate deformed nuclei. The axially symmetric deformation of the nuclear mean-field removes the degeneracy of spherical single-nucleon levels, and nucleons pairwise (spin up and down) occupy orbitals characterized by time-reversal degeneracy. For large deformations these levels can be labelled by a set of asymptotic Nilsson quantum numbers [26] and, because of the relatively weak Coulomb interaction in light nuclei, the localization of proton and neutron orbitals is similar in $\mathrm{Z}=\mathrm{N}$ nuclei. In the specific case of ${ }^{20} \mathrm{Ne}$, ten protons and ten neutrons occupy five deformed Nilsson levels, with the energy spacing between these levels being proportional to the deformation of the single-nucleon potential. Figure 2 shows the partial single-nucleon densities that correspond to highest occupied Nilsson orbital. Even without introducing a quantitative measure of localization, it is obvious that DD-ME2 predicts a much more localized density distribution. Similar results, i.e. more localized density distributions calculated with DD-ME2, are also obtained for the other four occupied orbitals.

Localization of densities that correspond to single-
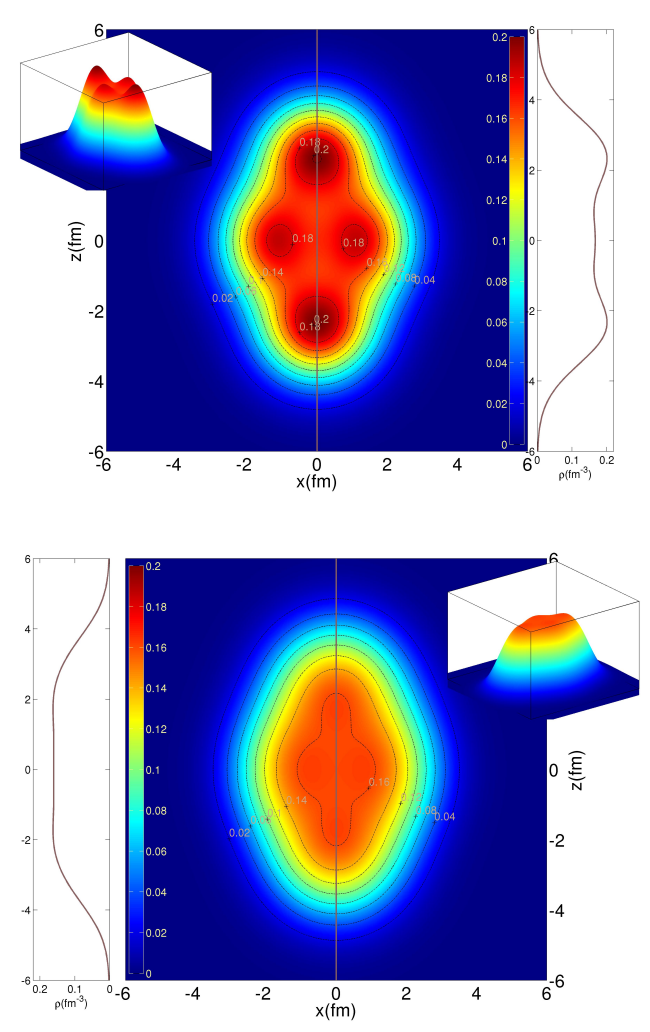

FIG. 1. Plots of self-consistent ground-state densities of ${ }^{20} \mathrm{Ne}$, calculated with the nuclear energy density functionals: DDME2 23] (top), and Skyrme SLy4 22, 30] (bottom). The densities (in units of $\mathrm{fm}^{-3}$ ) are plotted in the $\mathrm{x}-\mathrm{z}$ plane of the intrinsic frame of reference that coincides with the principal axes of the nucleus, with $\mathrm{z}$ chosen as the symmetry axis. The inserts show the corresponding three-dimensional density plots and the density profiles along the symmetry axis, respectively.

particle orbitals is a necessary precondition for the formation of clusters, and this effect can be traced back to the corresponding single-nucleon spectra. The comparison of spectra calculated with the two functionals shows that the one obtained with DD-ME2 is more spread out, and the more pronounced energy spacings between singleparticle levels are also reflected in the more localized wave functions and partial densities. Starting from degenerate spherical single-particle levels, the splitting of the corresponding Nilsson deformed states is proportional to the deformation and to the depth of the potential. Since the two functionals predict almost identical equilibrium deformations and radii for ${ }^{20} \mathrm{Ne}$, the different energy spacings in the single-nucleon spectra must reflect the difference in the corresponding potentials. In fact, the selfconsistent mean-field potential of DD-ME2 is considerably deeper than that of SLy4. In the centre of the nucleus the depth of the DD-ME2 single-neutron potential is $-78.6 \mathrm{MeV}$, whereas the depth of the SLy4 potential is $-69.5 \mathrm{MeV}$. The corresponding values of the single-proton 

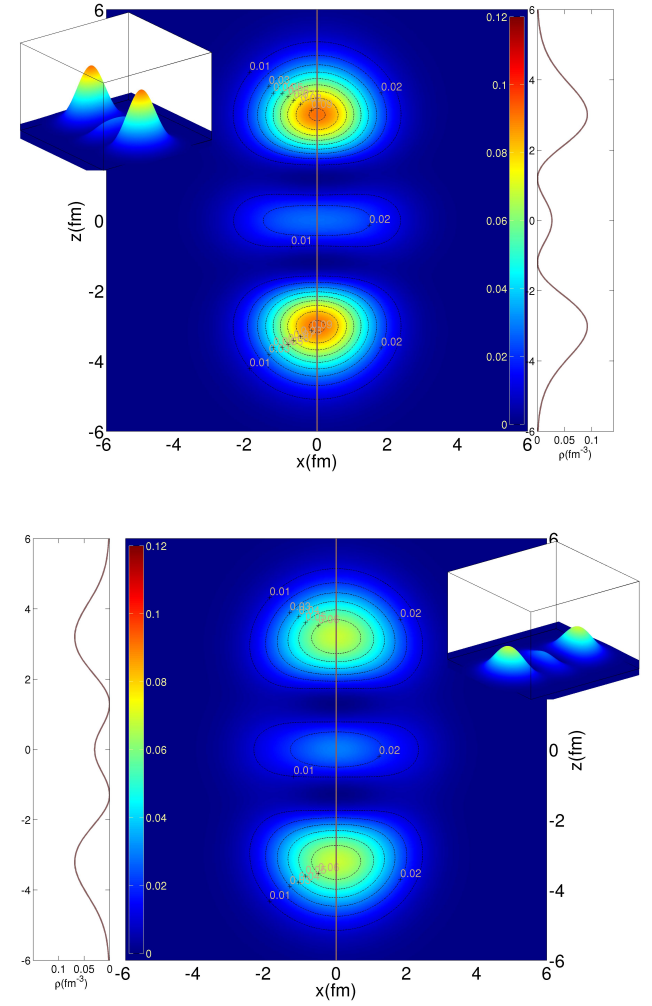

FIG. 2. Partial nucleon density distributions that correspond to highest occupied ( 2 protons spin up and down, and 2 neutrons spin up and down) in ${ }^{20} \mathrm{Ne}: \Omega^{\pi}[\mathrm{Nnz} \Lambda]=1 / 2^{+}[220]$, calculated with the nuclear energy density functionals: DDME2[23] (top), and SLy4 22, 30] (bottom).

potentials are: $-72.8 \mathrm{MeV}$ for DD-ME2, and $-64.6 \mathrm{MeV}$ for SLy4. The effect of the potential depth on the localization of wave functions is schematically illustrated in Fig. 3 where, as an approximation to nuclear potentials, we plot three harmonic oscillator potentials with different values of the depth: 30,45 and $60 \mathrm{MeV}$, but with the same radius $R=3 \mathrm{fm}$. The radial wave functions of the corresponding p-states are shown in the lower panel. The oscillator length $\mathrm{b}$ determines the position of the maximum and the dispersion of the wave function 27 . The deeper the potential, the smaller the oscillator length, and the wave functions become more localised. At the origin of clustering is, therefore, the depth of the selfconsistent single-nucleon mean-field potential associated with a given energy density functional. By performing a series of self-consistent mean-field calculations using a variety of non-relativistic and relativistic functionals, not only for ${ }^{20} \mathrm{Ne}$, but also for ${ }^{24} \mathrm{Mg},{ }^{28} \mathrm{Si}$ and ${ }^{32} \mathrm{~S}$, we have verified that pronounced cluster structures in deformed equilibrium shapes indeed occur only for deep single-nucleon potentials.

The difference of the potential depths calculated with DD-ME2 and SLy4 is characteristic for relativistic vs
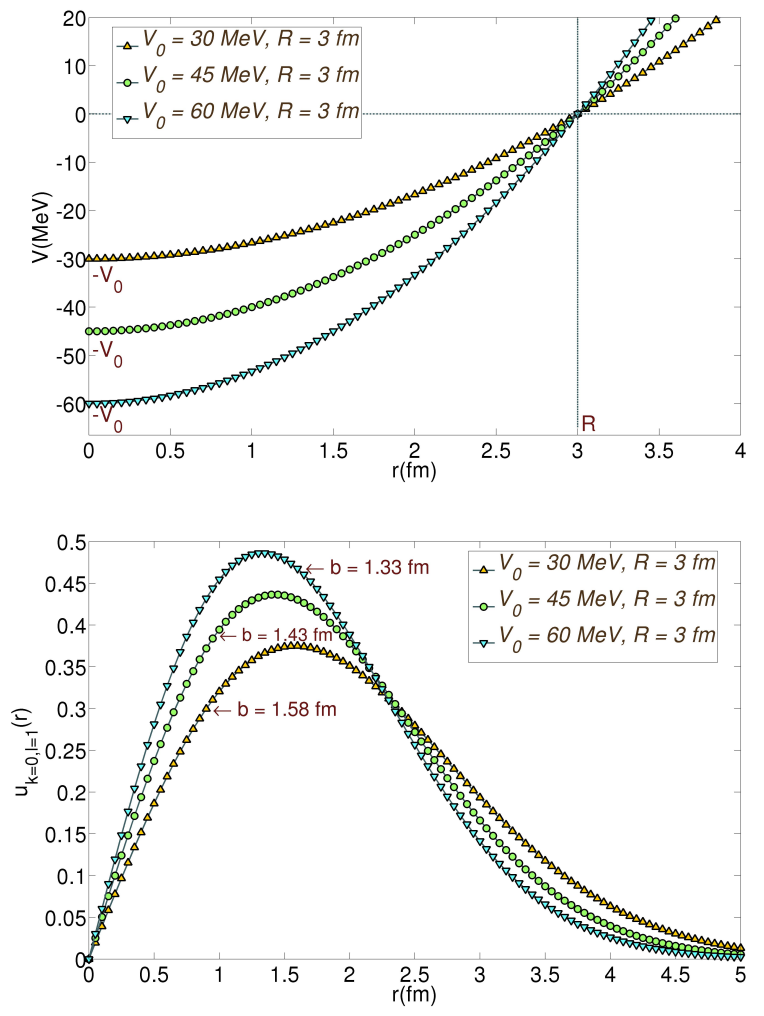

FIG. 3. Top: Harmonic oscillator potentials for three different values of the depth: 30,45 and $60 \mathrm{MeV}$, with the same radius $\mathrm{R}=3 \mathrm{fm}$. Bottom: the radial wave functions of the corresponding first p-state. The position of the maximum is determined by the oscillator length $b$.

non-relativistic self-consistent potentials. The depth of a relativistic potential is determined by the difference of two large fields: an attractive (negative) Lorentz scalar potential of magnitude $400 \mathrm{MeV}$, and a repulsive Lorentz vector potential $320 \mathrm{MeV}$ (plus the repulsive Coulomb potential for protons) [12 14. The choice of these potentials is further constrained by the fact that their sum $(\sim$ $700 \mathrm{MeV}$ ) determines the effective single-nucleon spinorbit potential. In a non-relativistic approach the spinorbit potential is included in a purely phenomenological way, with the strength of the interaction adjusted to empirical energy spacings between spin-orbit partner states. Since the relativistic scalar and vector fields determine both the effective spin-orbit potential and the self-consistent single-nucleon mean-field, for all relativistic functionals the latter is found to be deeper than the non-relativistic mean-field potentials, for which no such constraint arise.

More generally, fermionic systems can exhibit a crystalline phase or, on the other extreme, a quantum liquid phase. B. Mottelson considered the quantality [28] parameter to show that nuclear matter displays a quantum liquid structure. This concept can be generalised by con- 

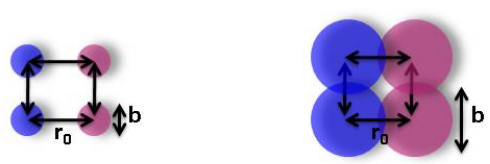

Crystal

Cluster

$\alpha \sim 1$

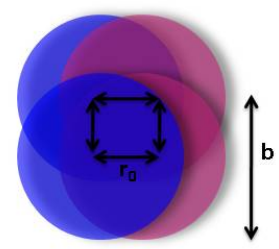

Quantum

liquid

$\alpha>1$

FIG. 4. Transition from crystalline to a quantum liquid phase, including the cluster phase. $\alpha=\mathrm{b} / \mathrm{r}_{0}$, where $\mathrm{b}$ is the dispersion of the fermion wave-function, and $r_{0}$ the typical interfermion distance.

sidering nuclear clusters as transitional states between crystalline and quantum liquid phases (Fig. 4). The dimensionless ratio $\alpha=\mathrm{b} / \mathrm{r}_{0}$, where $\mathrm{b}$ is the dispersion of the nucleon wave-function, and $\mathrm{r}_{0}$ the typical internucleon distance $(\sim 1.2 \mathrm{fm})$, is the natural parameter to quantify nuclear clustering, in analogy with similar considerations in condensed matter 29 .

For a harmonic oscillator

$$
\alpha=\frac{\sqrt{\hbar R}}{r_{0}\left(2 m V_{0}\right)^{1 / 4}}
$$

where $\mathrm{V}_{0}$ is the depth of the potential, and $\mathrm{R}$ the radius of the system.

When $\alpha>1$ nucleons are delocalised and the nucleus has a quantum liquid structure. The transition to a cluster state occurs when $\alpha \sim 1$, nucleons become more localised and form a molecular structure (Fig. 4). In the present analysis we find that $\alpha<1$ for the relativistic functional, whereas $\alpha>1$ for the non-relativistic functional. Moreover, from its definition in the case of a harmonic oscillator potential (see Eq. (1)), $\alpha$ obviously increases with the number of nucleons (nuclear radius). Cluster states, therefore, are less likely to appear in heavier nuclei.
[1] C.F.v Weizssacker, Naturwiss 26, 209-217 (1938)

[2] J.A. Wheeler, Phys. Rev. 52, 1107-1122 (1937).

[3] R. Tamagaki, H. Tanaka, Prog. Theor. Phys. 34, 191-192 (1965).

[4] W.v Oertzen, M. Freer, Y. Kanada-En'yo, Phys. Rep. 432, 43-113 (2006), and references therein.

[5] Y. Kanada En'yo, H. Horiuchi, Prog. Theor. Phys. Suppl. 142, 205-263 (2001).

[6] H. Feldmeier, K. Bieler, J. Schnack, Nucl. Phys. A 586, 493-532 (1995).

[7] T. Neff, H. Feldmeier, Nucl. Phys. A 713, 311-371 (2003).

[8] A. Tohsaki, H. Horiuchi, P. Schuck, G. Ropke, Phys. Rev. Lett. 87, 192501 (2001)

[9] H.O.U. Fynbo, et al., Nature 433, 136-139 (2005).

[10] H.J. Rose, G.A. Jones, Nature 307, 245-247 (1984).

[11] W. Greiner, J.Y. Park, W. Scheid, (World scientific, Singapore, 1995).

[12] G.A. Lalazissis, P. Ring, D. Vretenar, (Eds.), Lecture Notes in Physics 641, (Springer, Heidelberg 2004).

[13] M. Bender, P.-H. Heenen, P.-G. Reinhard, Rev. Mod. Phys. 75, 121-180 (2003).

[14] D. Vretenar, A.V. Afanasjev, G.A. Lalazissis, P. Ring, Phys. Rep. 409, 101-259 (2005).

[15] M. Girod, B. Grammaticos, Phys. Rev. C 27, 2317-2339 (1983).

[16] P. Arumugan, B.K. Sharma, S.K. Patra, Phys. Rev. C
71,064308 (2005).

[17] J.A. Maruhn, et al., Phys. Rev. C 74, 044311 (2006).

[18] T. Ichikawa, J.A. Marhun, N. Itagaki, S. Ohkubo, Phys. Rev. Lett. 107, 112501 (2011).

[19] T. Ichikawa, Y. Kanada En'yo, P. Moller, Phys. Rev. C 83, 054319 (2011).

[20] P.-G. Reinhard, J.A. Maruhn, A.S. Umar, V.E. Oberacker, Phys. Rev. C 83, 034312 (2011).

[21] L.M. Robledo, G.F. Bertsch, Phys. Rev. C 84, 054302 (2011).

[22] E. Chabanat, P. Bonche, P. Haensel, J. Meyer, R. Schaeffer, Nucl. Phys. A 635, 231-256 (1998).

[23] G.A. Lalazissis, T. Niksic, D. Vretenar, P. Ring, Phys. Rev. C 71, 024312 (2005).

[24] G. Fricke et al., Phys. Rev. C 45, 80-89 (1992).

[25] L. Chulkov et al., Nucl. Phys. A 603, 219-237 (1996).

[26] S.G. Nilsson, Mat Fys Medd Dan.Vid. Selsk. 29, 16 (1955).

[27] C. Cohen-Tannoudji, B. Diu, F. Laloe, Mecanique Quantique, (Hermann Ed., 1973).

[28] B. Mottelson, Nuclear Structure, Les Houches, Session LXVI, 25 (1996).

[29] D. Pines, P. Nozieres, The theory of quantum liquids, (Benjamin Inc., 1966).

[30] M.V. Stoitsov, J. Dobaczewski, W. Nazarewicz, P. Ring, Comp. Phys. Comm. 167, 43-63 (2005). 